$\mathrm{mg} / \mathrm{kg} /$ day for 33-81 months developed retinal pigmentation, hypopigmented retinal spots, and optic atrophy. Visual evoked potentials, performed in 21 , were abnormal initially in 2 and became abnormal during VGB therapy in 14. Electroretinography and electro-oculography were not available. (Koul R, Chacko A, Ganesh A, Bulusu S, Al Riyami K. Vigabatrin associated retinal dysfunction in children with epilepsy. Arch Dis Child Dec 2001;85:469-473). (Respond: Dr R Koul, Department of Child Health (Division of Paediatric Neurology), Sultan Qaboos University Hospital, Al Khod, PO Box 38, 123, Sultanate of Oman).

COMMENT. Regular eye examination including perimetry is recommended in patients treated with vigabatrin. Infants with epileptic syndromes and mental retardation, in whom perimetry is not appropriate, should receive funduscopic examination at least every 3 to 6 months, and retinography, electro-oculography, and VER when available. Retinal changes and visual field constriction associated with VGB can be irreversible, and risks may outweigh benefits if alternative less toxic treatments are available. (see Ped Neur Briefs Dec 2001;15:94-95).

\title{
X-LINKED MENTAL RETARDATION AND EPILEPSY SYNDROME
}

A kindred of 7 affected male infants with an X-linked mental retardation and epilepsy syndrome (XMRE), distinct from X-linked West and other mental retardation-epilepsy syndromes, is reported from the University of Michigan, Ann Arbor, MI. The locus for the new syndrome is identified on chromosome Xp21.1-p11.4. The tetraspanin gene, implicated in nonspecific mental retardation, was not involved. Seizures were primarily generalized, tonic-clonic, and atonic and began at 4 to 14 months of age (average age, 6.8 months). None had infantile spasms or hypsarrhythmia, and other than mental retardation and epilepsy affecting all patients, and mild rigidity and ataxia in 2, the neurologic examination was unremarkable. MRI, EMG, NCS, and laboratory metabolic tests were normal. (Hedera P, Alvarado D, Beydoun A, Fink JK. Novel mental retardation-epilepsy syndrome linked to Xp21.1-p11.4. Ann Neurol January 2002;51:45-50). (Respond: Dr John K Fink, 5214 Cancer Center Geriatrics Center Building, 1500 E Medical Center Drive, Ann Arbor, MI 48109).

COMMENT. X-linked mental retardation is clinically heterogeneous and is reported in $25 \%$ of all MR. The authors cite references to 202 XLMR genetically mapped syndromes and 33 identified XLMR genes. Seizures occur with several XLMR disorders, and West syndrome is sometimes transmitted as an X-linked disorder mapped to a locus on $\mathrm{Xp} 21.3-\mathrm{Xp} 22,1$, and distinct from the present novel family. In patients without recognizable metabolic or developmental brain abnormalities, a search for X-linked genetic factors is important in XMRE syndromes.

\section{AUTOSOMAL DOMINANT EPILEPSY SYNDROME LINKED TO 2p11}

A newly recognized autosomal dominant epilepsy syndrome with linkage to chromosome 2p11.1-q12.2 is described in an Italian pedigree of 8 patients reported from the Neurosciences Unit, Institute of Child Health and Great Ormond Street Hospital for Children, London, UK. Onset between ages 12 and 50 years, the syndrome is characterized by distal, semi-continuous rhythmic cortical myoclonus, generalized tonic-clonic and complex partial epileptic seizures (ADCME). The majority had only occasional seizures. Seizures were intractable in 3 who developed mild mental retardation. Valproate was most effective, while carbamazepine controlled seizures but exacerbated myoclonus. Interictal EEG abnormalities were generalized and focal frontotemporal. EMG studies of the 